\title{
El individuo y sus motivaciones en el proceso emprendedor
}

\author{
Flor Ángela Marulanda Valencia* \\ Iván Alonso Montoya Restrepo** \\ Juan Manuel Vélez Restrepo***
}

Fecha de recibido: 27 de octubre de 2017

Fecha de aprobado: 6 de junio de 2018

Para citar: Marulanda Valencia, F. A., Montoya Restrepo, I. A., \& Vélez Restrepo, J. M.

(2019). El Individuo y sus motivaciones en el proceso emprendedor.

Universidad E Empresa, 21(36), 149-174.

DOI: http://dx.doi.org/10.12804/revistas.urosario.edu.co/empresa/a.6197

* Doctora en Ingeniería, Industria y organizaciones de la Universidad Nacional de Colombia. Magíster en Ciencias de la Administración, Universidad EAFIT. Profesora Asociada de la Universidad Nacional de Colombia.

Correo electrónico: famarulan@unal.edu.co

** Doctor en Ciencias Económicas y magíster en Administración de la Universidad Nacional de Colombia. Profesor Titular de la Universidad Nacional de Colombia. Correo electrónico: iamontoyar@unal.edu.co

*** Doctor en Ingeniería y magíster en Ingeniería de la Universidad de São Paulo. Profesor Asociado de la Universidad Nacional de Colombia. Correo electrónico: jmvelez@unal.edu.co 


\section{Resumen}

Con el fin de identificar las motivaciones más importantes en el proceso de creación de empresa, en esta investigación se combinan aspectos cualitativos y cuantitativos. En primer lugar, se realizó un estudio exploratorio con emprendedores de Medellín a los que se les aplicó un cuestionario semiestructurado en el que se indagó, mediante pregunta abierta, por las motivaciones para crear y administrar su empresa. Con base en los resultados obtenigos y en la revisión de literatura se diseñó un constructo con escala tipo Likert, conformado por 6 dimensiones y 35 subdimensiones, que fue aplicado a otro grupo de emprendedores. Finalmente, se realizaron entrevistas en profundidad con algunos de los respondientes del último grupo. Se encontró que factores como el cumplimiento de un sueño, el crecimiento personal, los nuevos desafíos y la necesidad de independencia, fueron los más importantes para tomar la decisión, mientras que aspectos económicos y del entorno fueron menos significativos. Como contribución a la teoría de las motivaciones, entre otros, se propone romper con el paradigma de la clasificación tradicional entre emprendimiento por necesidad y emprendimiento de oportunidad.

Palabras clave: emprendimiento, emprendedor, motivaciones y creación de empresa.

\section{The Individual and its Motivations in the Entrepreneurship Process Abstract}

In order to identify the most important motivations in the process of company creation, this research combines qualitative and quantitative aspects. In the first place, an exploratory study was carried out with entrepreneurs from Medellin, to whom a semi-structured questionnaire was applied, in which an open question was asked about the motivations to create and manage their own company. Based on the results of this study, and the literature review, which is based more on theoretical contributions on human behavior and motivational aspects, rather than on more recent empirical developments, a construct with the Likert scale was designed, constituted by 6 dimensions and 35 subdimensions, which was applied to another group of entrepreneurs. Finally, in-depth interviews with some of the respondents of the latter group were conducted. Factors such as fulfillment of a dream, personal growth, new challenges and the need for independence were found to be the most important factors in making the decision; while economic and environmental aspects were less significant. As a contribution to the motivation theory, among others, the resulting proposal is to break away from the paradigm of the traditional classification between entrepreneurship by necessity and entrepreneurship of opportunity.

Keywords: Entrepreneurship, entrepreneur, motivations, business creation.

\section{O Indivíduo e suas motivações no processo empreendedor Resumo}

Com o objetivo de identificar as motivações mais importantes no processo de criação de empresa, nesta pesquisa combinam-se aspetos qualitativos e quantitativos. Em primeiro lugar, se realizou um estudo exploratório com empreendedores em Medellín aos que se aplicou um questionário semiestruturado no que se indagou, mediante pergunta aberta, pelas motivações ara criar e administrar sua empresa. Com base nos resultados deste, e a revisão de literatura, que se baseia mais em aportes teóricos sobre o comportamento humano e aspetos motivacionais, que em desenvolvimentos empíricos mais recentes, se desenhou um construto com escala tipo Likert, conformado por 6 dimensões e 35 subdimensões, que foi aplicado a outro grupo de empreendedores. Finalmente se realizaram entrevistas em profundidade com alguns dos respondentes do último grupo. Encontrou-se que fatores como o cumprimento de um sonho, o crescimento pessoal, os novos desafios e a necessidade de independência, foram os mais importantes para tomar a decisão. Enquanto os aspetos econômicos e do entorno, foram menos significativos. Como contribuição a teoria das motivações, entre outros, se propõe romper com o paradigma da classificação tradicional entre empreendimento por necessidade e empreendimento de oportunidade.

Palavras-chave: empreendimento, empreendedor, motivações, criação de empresa. 


\section{Introducción}

Promover el emprendimiento se ha convertido en un asunto de gran interés para los gobiernos, debido a su importancia en el desarrollo económico y social como mecanismo de generación de empleo e innovación, razón por la cual se crean una serie de organizaciones de promoción y apoyo a la creación de empresas a las que se destina una importante cantidad de recursos económicos. El problema es que la efectividad de las instituciones y los programas de promoción es muy reducido si se mide en términos de creación efectiva de empresa; en relación con el número de personas a las que se brinda acompañamiento y capacitación con este fin, las estadísticas de algunas instituciones de apoyo muestran que el porcentaje de empresas creadas en relación con el número de sensibilizaciones, esto es atención y capacitación a personas que llegan con ideas de emprendimiento, es inferior al 5 \% (Marulanda-Valencia, 2015). La situación se torna más preocupante cuando se analiza, además, la supervivencia de las mismas.

De acuerdo con Orrego (2008), estos pobres indicadores pueden explicarse en gran parte por la visión racionalista en el proceso de formación, con énfasis en la sostenibilidad económica, dejando de lado las razones culturales y humanas, relacionadas con el fortalecimiento del tejido social y la construcción de la sociedad. En este sentido, Shane, Locke y Collins (2003) sugieren que el desarrollo de la teoría empresarial requiere la consideración de las motivaciones de las personas que toman decisiones empresariales.

Precisamente, esta investigación se originó en el deseo de contribuir al reconocimiento de la importancia del individuo en el proceso del emprendimiento, enfocándose en el estudio de las motivaciones, por ser el primer paso en dicho proceso. Aunque se han realizado diversas investigaciones tendientes a encontrar las motivaciones de los emprendedores en diferentes países del mundo, los resultados de éstas no son universales, pues la cultura en la cual nos desarrollamos ejerce una gran influencia en todos los aspectos humanos (Hofstede, 1983; Shane et al., 2003), Circunstancia que puede verificarse con los resultados obtenidos por Kantis, Angelelli y Moori (2004) (tabla 1) en su investigación desarrollada para encontrar las motivaciones de los emprendedores en 13 países de América Latina, el este de Asia y el sur de Europa. A partir de dichos resultados, los autores plantean que las diferencias presentadas indicarían la existencia 
de contextos culturales muy diferenciados. Es así, que se hace necesario realizar un estudio que permita identificar las motivaciones que llevan a las personas del Valle de Aburrá a convertirse en emprendedoras, lo cual, además de contribuir con la teoría sobre el emprendimiento, servirá de base a las entidades y programas de fomento a esta actividad, para dirigir los esfuerzos y recursos hacia aquellos que tengan mayores posibilidades de crear y gestionar la empresa para que ésta permanezca.

La pregunta guía en el desarrollo de la misma fue ¿cuáles son los principales factores motivacionales que llevan a los emprendedores a crear y gestionar sus empresas?. Como población objetivo, se trabajó con los emprendedores pertenecientes al programa MIPES ${ }^{1}$ del SENA ${ }^{2}$, el cual tiene como objetivo apoyar el proceso de consolidación y formalización de micro y pequeñas empresas, especialmente aquellas que se han creado con el acompañamiento de la misma Institución.

Tabla 1. Principales motivaciones de los emprendedores dinámicos por país o región (lugar en el ranking y, entre paréntesis, porcentaje de emprendedores)

\begin{tabular}{llllllll}
\hline Motivación & $\begin{array}{l}\text { América } \\
\text { Latina }\end{array}$ & Italia & España & Japón & Taiwán & Corea & Singapur \\
\hline Autorrealización & $1^{\circ}(88)$ & $1^{\circ}(54)$ & $1^{\circ}(80)$ & $1^{\circ}(71)$ & $1^{\circ}(90)$ & $2^{\circ}(82)$ & $1^{\circ}(82)$ \\
\hline Contribuir a la sociedad & $3^{\circ}(58)$ & $7^{\circ}(15)$ & $4^{\circ}(41)$ & $2^{\circ}(61)$ & $3^{\circ}(68)$ & $1^{\circ}(83)$ & $3^{\circ}(71)$ \\
\hline Modelos de rol & $5^{\circ}(33)$ & $6^{\circ}(21)$ & $7^{\circ}(25)$ & $6^{\circ}(33)$ & $2^{\circ}(73)$ & $3^{\circ}(61)$ & $6^{\circ}(57)$ \\
\hline Independencia & $4^{\circ}(56)$ & $2^{\circ}(51)$ & $2^{\circ}(63)$ & $4^{\circ}(51)$ & $7^{\circ}(31)$ & $6^{\circ}(43)$ & $4^{\circ}(69)$ \\
\hline Mejorar ingresos & $2^{\circ}(74)$ & $3^{\circ}(42)$ & $3^{\circ}(50)$ & $3^{\circ}(52)$ & $5^{\circ}(54)$ & $4^{\circ}(56)$ & $2^{\circ}(77)$ \\
\hline Enriquecerse & $7^{\circ}(27)$ & $8^{\circ}(13)$ & $5^{\circ}(31)$ & $5^{\circ}(40)$ & $4^{\circ}(54)$ & $7^{\circ}(39)$ & $5^{\circ}(66)$ \\
\hline Seguir la tradición familiar & $8^{\circ}(19)$ & $4^{\circ}(40)$ & $8^{\circ}(23)$ & $9^{\circ}(6)$ & $8^{\circ}(10)$ & $8^{\circ}(10)$ & $8^{\circ}(29)$ \\
\hline Status social & $6^{\circ}(31)$ & $5^{\circ}(23)$ & $6^{\circ}(31)$ & $7^{\circ}(30)$ & $6^{\circ}(37)$ & $5^{\circ}(50)$ & $7^{\circ}(40)$ \\
\hline
\end{tabular}

Fuente: Kantis et. Al (2004).

El término "emprendedor" es un concepto muy amplio; para esta investigación se asumió la definición propuesta por Moriano et al. (2001): "La persona que pone en marcha una iniciativa empresarial, crea su propia empresa, solo o asociado con otros promotores,

1 Micros y Pequeñas Empresas.

2 Servicio Nacional de Aprendizaje. 
asumiendo los riesgos financieros que esto supone, aportando su trabajo y ocupándose de la dirección de la empresa” (p. 230).

El artículo presenta en primer lugar, un resumen de los planteamientos teóricos más utilizados en el estudio del emprendimiento desde el individuo y la forma como ha sido abordado el tema de las motivaciones; luego, se describen los aspectos metodológicos, los resultados y, antes de las conclusiones, se incluye un apartado de discusión de los resultados, en el que se analizan los hallazgos a la luz de la literatura.

\section{Revisión de literatura}

El concepto de "motivación para emprender" ha surtido un sustancial avance que parte desde una concepción puramente económica, hasta considerarle como un proceso multidimensional en el que intervienen aspectos personales, sociales y culturales, gracias a las teorías que sobre el comportamiento humano se han desarrollado desde la psicología y la sociología. A continuación, se presenta un resumen de las teorías que se tuvieron en cuenta.

\subsection{Teoría del Comportamiento Planificado}

Esta teoría, reconocida como TPB (Theory of Planned Bebavior), fue desarrollada por Icek Ajzen (1991) con el fin de explicar la intención del individuo para realizar una conducta determinada. Las intenciones reflejan los factores motivacionales que influyen en un comportamiento y son indicaciones del nivel de esfuerzo que las personas están dispuestas ejercer, con el fin de realizar la conducta. El autor explica que son tres los determinantes, conceptualmente independientes, de la intención. El primero es la actitud hacia el comportamiento, que está dado por el grado en que una persona tiene una valoración favorable o desfavorable de la conducta en cuestión. El segundo es un factor social que el autor denomina norma subjetiva, y se refiere a la percepción de la presión social para realizar o no la conducta. El tercer determinante es el grado de control conductual percibido, que está relacionado con la facilidad o dificultad percibida de la realización de la conducta. El autor establece diferencias entre este concepto y el de "locus de control interno", el cual se refiere a que las personas asumen la responsabilidad de sus decisiones y actuaciones, mientras que este es muy compatible con el de “autoeficacia” en la forma como lo plantea Bandura (1977). 


\subsection{Teoría del Evento Empresarial}

Albert Shapero (1985), se refiere al "evento empresarial" como el proceso de formación de empresa, producto de dos decisiones: la decisión de un cambio de vida y la decisión de iniciar una compañía. La decisión de cambio de vida surge de la desestabilización que generan en las personas diferentes situaciones, sean negativas o positivas (Shapero, 1985, p. 25). Sin embargo, una vez se presenta la situación motivadora (negativa o positiva), el interrogante que surge es ¿qué es lo que hace que unas personas creen empresas y otras no? Como respuesta, el autor explica que la decisión de crear empresa es el producto de dos percepciones: conveniencia o deseabilidad y factibilidad o viabilidad.

Las percepciones de conveniencia tienen que ver con los valores y son resultado de la cultura, estructura socioeconómica, familia, educación, pares y personas influyentes. Afirma que son más propensas a la creación de empresas aquellas culturas que valoran los comportamientos asociados a los negocios, como la independencia, la innovación y la toma de riesgos. Dentro de los ejemplos que cita, incluye regiones como Smoeland en Suiza, Cork en Irlanda y Antioquia en Colombia (Shapero, 1985, p. 26). También destaca la influencia de la familia y explica que son más proclives a crear su propia empresa los hijos de empresarios, de profesionales independientes, de artesanos o de agricultores. Igualmente, pueden ejercer incidencia otras personas con las que el emprendedor se identifica, como profesores, mentores, figuras públicas y escritores. Si bien los planteamientos de Shapero son anteriores a la Teoría del Comportamiento Planificado de Ajzen, puede afirmarse que el concepto de conveniencia está muy asociado con el de normas subjetivas.

En cuanto a la percepción de factibilidad, esta es considerada por el autor como una mezcla de conocimientos, recursos, garantías y experiencia obtenida en un negocio particular o en la educación formal, por lo que muchos emprendedores crean empresas en sectores en los cuales han laborado. También mejora la percepción de factibilidad el haber tenido contacto con clientes y un acercamiento al mercado, pero para el autor la mayor influencia proviene de la observación de otros: ver que otros similares a uno hacen ciertas cosas es más motivador que simplemente imaginarse a uno mismo haciéndolas (Shapero, 1985). 
Otros desarrollos sobre el tema han consistido en clasificar las motivaciones en una forma dualista. De éstas, la más generalizada, por ser la que utiliza el GEm (Global Entrepreneurship Monitor), es la diferenciación entre emprendimiento por necesidad y emprendimiento de oportunidad. Se considera que una persona que emprende lo hace por solo una de dos razones: la detección de una oportunidad que le lleva a crear empresa como elección entre varias opciones de carrera para su vida (se denota como OEA, sigla en inglés de Opportunity Entrepreneurship Activity), mientras que otras personas emprenden un negocio porque no encuentran otra forma para subsistir (se conoce como NEA, de Necessity Entrepreneurship Activity). Otras clasificaciones dicotómicas de las motivaciones son fatores endógenos vs. factores exógenos, y factores de empuje (push) vs. factores de atracción (pull) (Marulanda-Valencia, Montoya \& Vélez, 2014 B).

\subsection{Categorías y dimensiones}

Debido a que no siempre se pueden posicionar las motivaciones en una de las clasificaciones dualistas, muchos investigadores simplemente listan estas motivaciones. A medida que los estudios se van tornando más sofisticados, se han ido estableciendo una serie de categorías que dan cuenta de la complejidad del tema.

Para la realización de este estudio, se tomó como base la propuesta de Morales-Gualdrón, Gutiérrez-Gracia y Roig-Dobón (2009), que consiste en un constructo basado en seis dimensiones: personal, oportunidad, conocimiento científico, disponibilidad de recursos, organización incubadora y entorno social, y 12 subdimensiones. El modelo fue aplicado por los autores para investigar las motivaciones de los emprendedores académicos en España.

\section{Metodología}

Teniendo en cuenta que, como lo demostró Kantis (2004), las motivaciones cambian de acuerdo con el contexto, y en este caso, en relación con el estudio de Morales et. al, se presentaba diferencia tanto en la población objetivo (empredimiento tradicional vs. emprendimiento académico), así como en los aspectos culturales (España vs. Colombia), se construyó un instrumento con base en la revisión de la literatura y en un estudio exploratorio previo. Para el estudio exploratorio se utilizó un cuestionario de preguntas abiertas, que se aplicó a un grupo de 18 emprendedores del Valle de Aburrá, quienes 
expresaban abiertamente todos los factores motivacionales que influyeron en su decisión de crear y administrar su empresa.

El constructo definitivo, que se llevó a una escala tipo Likert, está compuesto por seis dimensiones y 35 subdimensiones, de las cuales se hace una breve presentación a continuación:

\subsection{Motivaciones de tipo personal}

En esta categoría se incluyen los factores de orden psicológico y de bienestar laboral, incluyendo el aspecto económico. Motivaciones como la necesidades de logro e independencia y el deseo de riqueza pueden ser decisivas en la determinación de convertirse en emprendedor (Autio \& Kauranen, 1994), también incluye aspiraciones como cumplir un sueño, alcanzar retos y el crecimiento personal, ya que el deseo de superación y progreso lleva a los individuos a buscar siempre un estadio superior en sus actividades (Varela \& Bedoya, 2006).

Para autores como Shapero (1985), por otro lado, la motivación dominante para convertirse en emprendedor es la necesidad de independencia. El término se interpreta desde varios puntos de vista: la posibilidad de tomar sus propias decisiones y asumir la responsabilidad que ello implica (Carter et al., 2003; Raposo et al., 2008; Sane et al.,2003), y la autonomía y flexibilidad en el manejo del tiempo (Manolova, Brush \& Edelman, 2008).

Por su parte, los factores de tipo económico, han sido considerados uno de los objetivos principales de los emprendedores (Raposo et al., 2008) e incluyen desde el deseo de ganar seguridad para sí mismo, el cónyuge e hijos, y contribuir al bienestar de los familiares (Carter, Gartner, Shaver, \& Gatewood, 2003), hasta el de lograr “éxito financiero” (Edelman, Brush, Manolova \& Greene, 2010).

\subsection{Motivaciones relacionadas con el conocimiento}

Esta categoría incluye desde las aptitudes previas que un individuo tiene sobre cierta materia, adquiridas mediante una experiencia laboral, que le capacitan, incluso, para detectar oportunidades (Shepherd, 2005), hasta el conocimiento técnico y la innovación, producto de la investigación y que motivan al emprendedor a estar a la vanguardia en nuevas tecnologías y continuar aprendiendo (Carter, Gartner, Shaver \& Gatewood, 2003). 


\subsection{Motivaciones relacionadas con la oportunidad}

De acuerdo con Shane y Venkataraman (2000), una oportunidad puede convertirse en una actividad rentable para un individuo, solo si él o ella reconocen que existe y tiene valor.

\subsection{Motivaciones relacionadas con la disponibilidad de recursos}

Paturel (1997) planteó tres categorías de recursos necesarios para crear una empresa, así: los recursos financieros, los recursos técnicos y logísticos y, la red de relaciones. En estudios más recientes se ha reconocido que la visión que el potencial emprendedor tiene de sí mismo y de sus capacidades para llevar a cabo el proyecto es un recurso muy importante (Segal, Borgia \& Schoenfeld, 2005; Farmer, Yao \& Kung-Mcintyre, 2011), este aspecto se refiere al concepto autoeficacia percibida, desarrollado por Albert Bandura (1977). También se ha incluido en esta categoría los apoyos públicos que se brindan como fomento al emprendimiento, y las redes de relaciones de posibles clientes y proveedores.

\subsection{Motivaciones relacionadas con la experiencia laboral}

Este aspecto hace referencia a las vivencias del emprendedor en su vida laboral, antes de crear su empresa, e influye en el proceso de fundación de diferentes maneras: crear un negocio en el mismo sector económico o que la pérdida del empleo sea el hecho detonante.

\subsection{Motivaciones relacionadas con el entorno social}

Se incluyen en esta categoría, de un lado, la influencia que la familia, los amigos, las personas a las que se admira y el ejemplo de compañías exitosas tienen sobre el emprendedor y su decisión de crear empresa. De otro lado, se evalúa el impacto de las organizaciones y las campañas de fomento al emprendimiento. Por último, se tienen en cuenta los valores sociales, que se refieren al deseo del emprendedor de contribuir con la comunidad (Kantis, Angelelli \& Moori, 2004). Por ejemplo, en la biotecnología, la búsqueda de una cura para una enfermedad puede ser un motivador más poderoso que la creación de riqueza personal (Carsrud \& Brännback, 2011), o puede ser a un nivel más general, el interés de generar empleo y contribuir al desarrollo del país. 


\section{Recolección de la información}

Considerando que el emprendimiento se ha constituido en un elemento importante de política pública, se buscó el apoyo del SENA, entidad colombiana que tiene, posiblemente, el programa más completo de fomento al emprendimiento en todo el país, pues cubre todas las etapas del proceso, desde el acompañamiento a la persona que manifiesta su deseo de ser emprendedor, pasando por la financiación y ayuda en la puesta en marcha, hasta la capacitación cuando las empresas están consolidadas. Concretamente, el programa MIPES ha buscado el fortalecimiento de las micro y pequeñas empresas formalizadas $^{3}$ (SENA, 2012). Se eligió esta población por el apoyo institucional ofrecido por el SENA a la investigación y la posibilidad de identificar a los empresarios que cumplen con el perfil definido.

La base de datos proporcionada por el SENA, compuesta por 82 empresas, fue depurada de acuerdo con la definición de emprendedor y otros criterios como antigüedad superior a diez años, ya que este es el tiempo aproximado que llevan los programas de fomento, cuyo impacto es uno de los factores que se evalúa. También, se excluyeron las empresas que no son gestionadas por sus creadores, las que son administradas por extranjeros y las famiempresas. De esta forma, se obtuvo una base de datos depurada de 44 emprendedores.

Para la recolección de la información se utilizó un formulario de escala tipo Likert que evalúa las categorías de la motivación descritas en el apartado anterior. Se realizó contacto telefónico con cada uno de los emprendedores de la base de datos depurada, solicitándole diligenciar el formulario. Dadas las ocupaciones de éstos, fue necesario insistir durante tres meses, vía telefónica y correo electrónico, para lograr las respuestas. Además del envío vía internet, se ofreció la visita personal a los emprendedores. En total se lograron 35 respuestas válidas, tres de ellas con visita personal.

\subsection{Procesamiento de la información}

Teniendo en cuenta que no se encontraron investigaciones de este tipo en el país, se hace énfasis en cuáles son las motivaciones más importantes para las personas que deciden crear y administrar sus empresas. Por esta razón, se presenta con un análisis

3 Para el SENA se consideran formalizadas las empresas que, además de los aportes a la seguridad social, realizan aportes parafiscales. 
descriptivo de los resultados, tanto de la información demográfica como de las motivaciones. En el apartado Discusión se analizan los resultados a la luz de la literatura. Para el procesamiento de los datos se utilizó el software SPSS.

\section{Resultados}

\subsection{Descripción demográfica}

De acuerdo con la tabla 2, puede hablarse de un perfil del emprendedor MIPES como mayor de 25 años, profesional, casado y con hijos. En cuanto a las empresas, el $83 \%$ son micro, en su mayoría tienen entre uno y seis años de funcionamiento, y pertenecen al sector de servicios y, en menor medida, a las manufacturas.

Tabla 2. Perfil demográfico del emprendedor MIPES

\begin{tabular}{|c|c|}
\hline Criterio & Participación \\
\hline \multicolumn{2}{|c|}{ Sexo } \\
\hline Femenino & $51 \%$ \\
\hline Masculino & $49 \%$ \\
\hline \multicolumn{2}{|c|}{ Edad } \\
\hline Menos de 25 años & $6 \%$ \\
\hline Entre 25 y 35 años & $34 \%$ \\
\hline Entre 35 y 45 años & $29 \%$ \\
\hline Entre 45 y 55 años & $31 \%$ \\
\hline \multicolumn{2}{|c|}{ Educación } \\
\hline Bachillerato & $3 \%$ \\
\hline Técnica & $14 \%$ \\
\hline Tecnología & $23 \%$ \\
\hline Universitaria completa & $51 \%$ \\
\hline Especialización & $9 \%$ \\
\hline
\end{tabular}




\begin{tabular}{|c|c|}
\hline Criterio & Participación \\
\hline \multicolumn{2}{|c|}{ Estado Civil } \\
\hline Casado & $57 \%$ \\
\hline Soltero & $34 \%$ \\
\hline Otro (unión libre, divorciado) & $9 \%$ \\
\hline \multicolumn{2}{|c|}{ Número de hijos } \\
\hline 0 & $40 \%$ \\
\hline 1 & $23 \%$ \\
\hline 2 & $26 \%$ \\
\hline 3 & $11 \%$ \\
\hline
\end{tabular}

Fuente: elaboración propia.

\subsection{Motivaciones}

Uno de los métodos más utilizados para medir la confiabilidad de un constructo es el Alfa de Cronbach. ${ }^{4}$ Para considerarla adecuada debe dar como resultado al menos 0,7. En este caso el Alfa fue de 0,843 , por tanto se puede afirmar que el instrumento utilizado para evaluar las motivaciones del emprendedor MIPES es confiable.

En la tabla 3, se presenta un resumen de los resultados obtenidos, indicando la importancia relativa para cada una de las subdimensiones, que también pueden observarse en la figura 1. De la tabla puede observarse cuáles fueron las motivaciones más importantes así:

- De tipo personal: Las motivaciones que tuvieron una importancia relativa superior al $90 \%$ (suma de respuestas "importante" y "muy importante") son "cumplir con un sueño o meta personal", "alcanzar nuevos retos personales" y "autonomía e independencia en el trabajo". El aspecto económico, aunque es importante, no es lo primordial, el factor "Tener un medio de subsistencia" fue el más influyente.

- Conocimiento: En esta dimensión, la variable más importante fue "explotar comercialmente el conocimiento y la experiencia personal”.

4 Es un coeficiente que permite evaluar la consistencia interna de la prueba (Cervantes, 2005). La interpretación de los resultados que arroja esta prueba es que cuanto más se acerque el índice al extremo 1, mejor es la fiabilidad. 
- Oportunidad: La detección de una necesidad insatisfecha es el componente que fue considerado más influyente de esta categoría, aunque su importancia relativa fue de solo $77 \%$.

- Disponibilidad de recursos: El recurso que los encuestados encontraron más importante fue el de sus "habilidades emprendedoras". También fueron importantes "red de contactos en el mercado" y "conocimiento de posibles proveedores". En cuanto a los medios económicos, predominó "invertir los recursos propios o de la familia”, mientras que la financiación externa tuvo muy poca participación.

- Experiencia laboral: Los dos factores que componen esta dimensión: "inconformidad con la situación laboral cuando era empleado" y "terminación de vinculación laboral y oportunidad de continuar en empresa propia” fueron medianamente importantes para los emprendedores.

- Entorno social e institucional: Seguir el ejemplo de empresas o proyectos exitosos fue uno de los factores importantes de esta dimensión.

Tabla 3. Importancia relativa de cada una de las motivaciones para emprender

\begin{tabular}{|c|c|c|c|}
\hline & Dimensiones & $\begin{array}{c}\text { Variables relativas a las motivaciones de } \\
\text { tipo personal }\end{array}$ & $\begin{array}{l}\text { Importancia } \\
\text { Relativa* }\end{array}$ \\
\hline M & \multirow{9}{*}{ De tipo personal } & Cumplir con un sueño o meta personal. & $92 \%$ \\
\hline 0 & & Alcanzar nuevos retos personales. & $94 \%$ \\
\hline $\mathbf{T}$ & & $\begin{array}{l}\text { Vivir una experiencia que me permita crecimiento } \\
\text { personal. }\end{array}$ & $86 \%$ \\
\hline I & & Tener autonomía e independencia en mi trabajo. & $91 \%$ \\
\hline V & & La mejora del entorno de trabajo personal. & $77 \%$ \\
\hline A & & Tener mayor flexibilidad en el uso del tiempo. & $63 \%$ \\
\hline C & & Tener un medio de subsistencia. & $82 \%$ \\
\hline I & & Obtener altos ingresos económicos. & $71 \%$ \\
\hline 0 & & Construir un negocio familiar. & $54 \%$ \\
\hline $\mathbf{N}$ & \multirow{3}{*}{$\begin{array}{l}\text { Relacionadas con el } \\
\text { conocimiento }\end{array}$} & $\begin{array}{l}\text { Explotar comercialmente el conocimiento y la } \\
\text { experiencia personal. }\end{array}$ & $86 \%$ \\
\hline $\mathbf{E}$ & & $\begin{array}{l}\text { Explotar comercialmente una innovación } \\
\text { generada a partir de resultados de investigación. }\end{array}$ & $54 \%$ \\
\hline $\mathbf{S}$ & & $\begin{array}{l}\text { Explotar comercialmente una innovación } \\
\text { generada a partir del conocimiento y la tecnología } \\
\text { provisto por otros. }\end{array}$ & $46 \%$ \\
\hline
\end{tabular}




\begin{tabular}{|c|c|c|c|}
\hline & Dimensiones & $\begin{array}{l}\text { Variables relativas a las motivaciones de } \\
\text { tipo personal }\end{array}$ & $\begin{array}{l}\text { Importancia } \\
\text { Relativa* }\end{array}$ \\
\hline & \multirow{3}{*}{ Oportunidad } & La detección de una necesidad insatisfecha. & $77 \%$ \\
\hline $\mathbf{P}$ & & El descubrimiento de un nuevo producto/servicio. & $49 \%$ \\
\hline A & & El desarrollo de un nuevo método de producción. & $32 \%$ \\
\hline $\mathbf{R}$ & \multirow{9}{*}{$\begin{array}{l}\text { Disponibilidad de } \\
\text { recursos }\end{array}$} & La posibilidad de financiación externa. & $29 \%$ \\
\hline \multirow[t]{2}{*}{ A } & & $\begin{array}{l}\text { Responder a convocatorias externas (públicas y/o } \\
\text { privadas). }\end{array}$ & $23 \%$ \\
\hline & & $\begin{array}{l}\text { Invertir los recursos financieros propios o de mi } \\
\text { familia. }\end{array}$ & $69 \%$ \\
\hline $\mathbf{E}$ & & La existencia de apoyos públicos. & $15 \%$ \\
\hline M & & $\begin{array}{l}\text { La disponibilidad de una red de contactos en el } \\
\text { mercado potencial. }\end{array}$ & $57 \%$ \\
\hline $\mathbf{P}$ & & $\begin{array}{l}\text { Poner en práctica las habilidades emprendedoras } \\
\text { que posee. }\end{array}$ & $88 \%$ \\
\hline $\mathbf{R}$ & & Conocimiento de posibles proveedores. & $63 \%$ \\
\hline $\mathbf{E}$ & & $\begin{array}{l}\text { Políticas públicas y estímulos para el } \\
\text { emprendimiento. }\end{array}$ & $20 \%$ \\
\hline $\mathbf{N}$ & & Acompañamiento externo público y/o privado. & $34 \%$ \\
\hline D & \multirow{2}{*}{ Experiencia laboral } & $\begin{array}{l}\text { Inconformidad con la situación laboral cuando era } \\
\text { empleado. }\end{array}$ & $55 \%$ \\
\hline $\mathbf{E}$ & & $\begin{array}{l}\text { terminación de vinculación laboral y oportunidad } \\
\text { de continuar en empresa propia. }\end{array}$ & $46 \%$ \\
\hline \multirow[t]{9}{*}{$\mathbf{R}$} & \multirow{9}{*}{ Entorno social } & $\begin{array}{l}\text { Continuar con una tradición familiar de } \\
\text { emprendedores. }\end{array}$ & $34 \%$ \\
\hline & & La influencia de los amigos. & $23 \%$ \\
\hline & & $\begin{array}{l}\text { Seguir el ejemplo de empresas o proyectos } \\
\text { exitosos. }\end{array}$ & $65 \%$ \\
\hline & & Lograr un mejor status social. & $37 \%$ \\
\hline & & $\begin{array}{l}\text { Existencia de organizaciones de fomento al } \\
\text { emprendimiento. }\end{array}$ & $26 \%$ \\
\hline & & $\begin{array}{l}\text { Las campañas de fomento a la creación de } \\
\text { empresas. }\end{array}$ & $25 \%$ \\
\hline & & $\begin{array}{l}\text { La actitud existente en el entorno hacia la creación } \\
\text { de empresas. }\end{array}$ & $49 \%$ \\
\hline & & Generar empleo. & $80 \%$ \\
\hline & & Contribuir al bienestar de la comunidad. & $80 \%$ \\
\hline
\end{tabular}

Fuente: elaboración propia. 


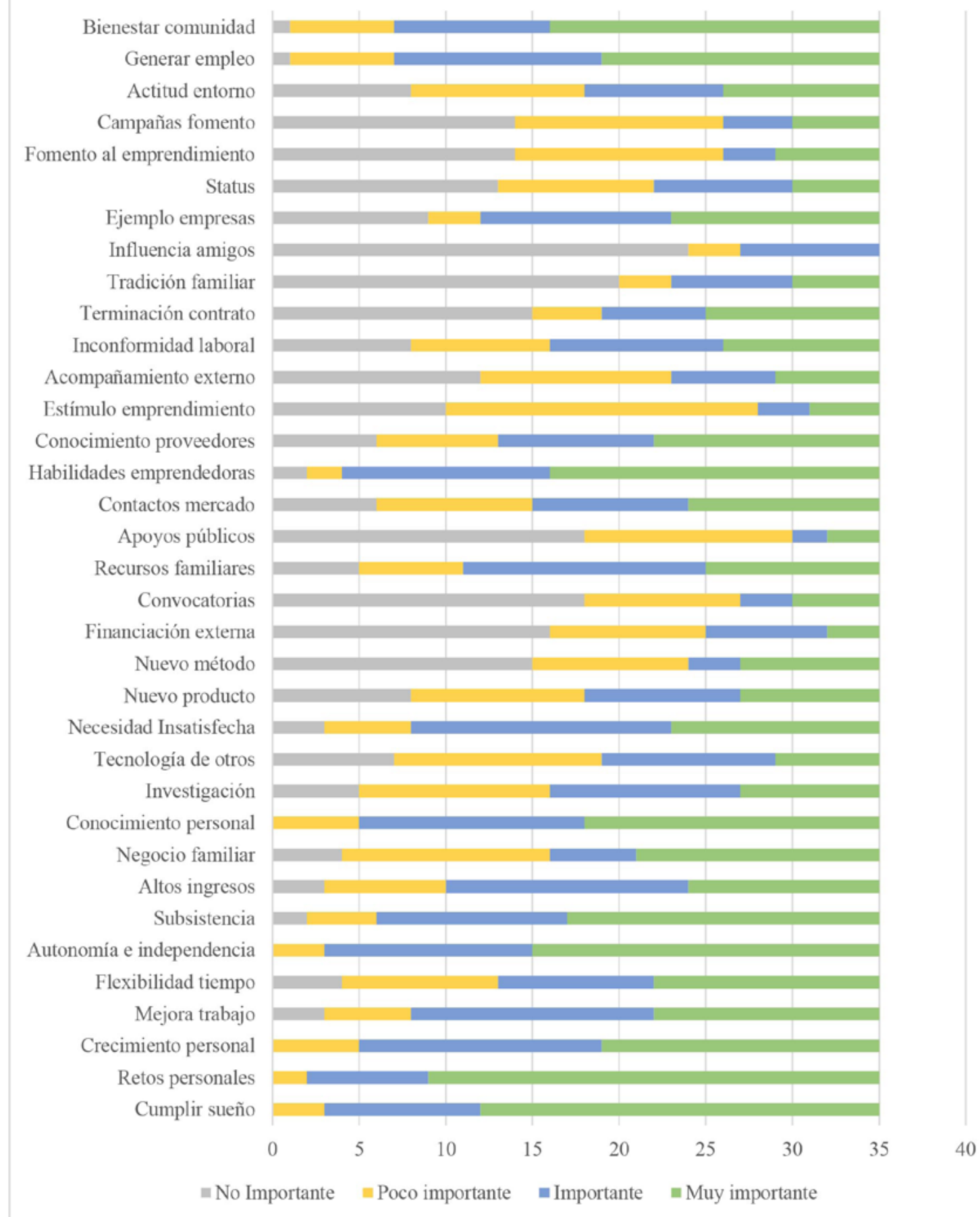

Figura 1. Motivaciones de los emprendedores MIPES para crear y gestionar sus empresas Fuente: elaboración propia. 


\section{Discusión de los resultados}

El perfil demográfico del emprendedor MIPES es un indicador de que la carrera que ha escogido, en este caso, administrar la empresa que ha creado, es parte de su proyecto de vida o como lo expresa Shapero (1985) es el camino de vida que ha decidido seguir.

En la tabla 4 se presenta un compendio de los factores que tuvieron una importancia relativa superior al $80 \%$. En primer lugar, con una importancia superior al $90 \%$ se encuentran "alcanzar nuevos retos personales", "cumplir con un sueño o meta personal" y autonomía e independencia en el trabajo", que son los que conforman la "motivación de logro" o "necesidad de logro", Nach (McClelland, 1961). En general, al revisar todos los factores que componen la motivación para emprender, se encuentra que los aspectos más internos o los llamados "factores endógenos" (Quevedo, Izar \& Romo, 2010), o "aspiraciones personales" (García, 1992) son los que más influyen en la decisión de crear y administrar la propia empresa, lo cual es coherente con el planteamiento teórico de este trabajo, en cuanto a la influencia de la individualidad en el tema del emprendimiento.

Tabla 4. Factores motivacionales más importantes para los emprendedores MIPES

\begin{tabular}{ll}
\multicolumn{1}{c}{ Factor } & $\begin{array}{c}\text { Importancia Relativa } \\
\text { (\%) }\end{array}$ \\
\hline Alcanzar nuevos retos personales. & 94 \\
\hline Cumplir con un sueño o meta personal. & 91 \\
\hline $\begin{array}{l}\text { Tener autonomía e independencia en mi } \\
\text { trabajo. }\end{array}$ & 91 \\
\hline $\begin{array}{l}\text { Poner en práctica las habilidades } \\
\text { emprendedoras que posee. }\end{array}$ & 89 \\
\hline $\begin{array}{l}\text { Vivir una experiencia que me permita } \\
\text { crecimiento personal. }\end{array}$ & 86 \\
\hline $\begin{array}{l}\text { Explotar comercialmente el conocimiento } \\
\text { y la experiencia personal. }\end{array}$ & 86 \\
\hline $\begin{array}{l}\text { Tener un medio de subsistencia. } \\
\text { Generar empleo. }\end{array}$ & 83 \\
\hline \begin{tabular}{l} 
Contribuir al bienestar de la comunidad. \\
\hline
\end{tabular} & 80 \\
\hline
\end{tabular}

Fuente: elaboración propia. 
$\mathrm{Al}$ analizar estos resultados, de acuerdo con la teoría de las necesidades de Maslow (1991), se halla que el grupo cinco: "el deseo de fuerza, logro, adecuación, maestría y competencia, confianza ante el mundo, independencia y libertad" y el seis "necesidad de autorrealización" corresponden prácticamente a los factores más importantes para los emprendedores MIPES. Cabe recordar que sobre este último grupo, el autor afirma que así se tengan cubiertas todas las demás, siempre habrá una inquietud en la persona, "a menos que esté haciendo aquello para lo que él individualmente esté capacitado", en otras palabras "lo que los humanos pueden ser, es lo que deben ser" (Maslow, 1991, p. 32).

Si se analizan estos resultados a la luz de la teoría del Comportamiento Planificado de Ajzen (1991), en cuanto a los determinantes de la intención, estos permiten establecer que los emprendedores tienen una valoración favorable sobre esta actividad, pues es el primer paso que dan hacia este camino, es la decisión de convertirse en tales, para ellos es una opción de carrera deseable por las satisfacciones personales que puede ofrecerles; como lo expone Rodríguez, "la noción de "placer" es indisociable de los procesos de motivación y emprendimiento" (2009, p. 113).

Otro determinante de la actividad emprendedora a la luz de los planteamientos de Ajzen es el grado de control percibido sobre la conducta, que está relacionado con la facilidad o dificultad percibida de la realización de la misma y que es compatible con el concepto de "autoeficacia" de Bandura (1977), en cuanto a que el comportamiento está influenciado por la confianza que tienen las personas en su capacidad para llevarlo a cabo. En este caso, "poner en práctica las habilidades emprendedoras que posee" es el recurso más importante en el proceso de creación y administración de su negocio. Sobre este aspecto, Manolova et al. (2008) explica que un emprendedor estará dispuesto a realizar todos los esfuerzos para iniciar una empresa si considera que tiene las capacidades para que esta sea exitosa. A la luz de los planteamientos de la Teoría del evento empresarial (Shapero, 1985), este es un recurso que incrementa la percepción de viabilidad o factibilidad.

Otros recursos que contribuyen a mejorar la percepción de viabilidad y que son importantes, aunque no tan relevantes, para los emprendedores MIPES son "la disponibilidad de una red de contactos en el mercado potencial" y "conocimiento de posibles proveedores". Gran parte de estos contactos se adquieren en la experiencia laboral previa a la creación de la empresa, 
como también puede ser el fruto de la observación y la investigación que el emprendedor realiza. Cualquiera que sea el medio, a los emprendedores les da confianza el haber tenido contacto con el mercado, tanto de fuentes de aprovisionamiento como de clientes.

El tercer determinante considerado por Ajzen es el factor social o norma subjetiva, que se refiere a la percepción de la presión social para realizar o no realizar la conducta y está muy relacionado con el concepto de deseabilidad o conveniencia planteado por Shapero, que lo explica como la valoración que se da en el entorno a la actividad emprendedora. En el caso MIPES, ni la familia, ni los amigos, ni el status se consideran motivadores importantes. En forma similar, la imagen de la sociedad sobre la actividad emprendedora tampoco es trascendental para ellos; esto podría deberse a que la tradición de Antioquia en esta actividad lleva a que esta no sea una preocupación para la persona que quiere tener su propio negocio, es decir, se da por descontado que esto no está mal visto, sino que, al contrario, es un comportamiento deseable. Esto puede sustentarse en el hecho de que los factores "generar empleo" y "contribuir al bienestar de la comunidad" son los más importantes de la dimensión "entorno social”. Así, la actividad que ellos desarrollan es una forma de ayudar a mitigar la problemática del desempleo y de ofrecer soluciones directas a necesidades específicas con el desarrollo del objeto social del negocio, en forma ética.

En relación con el aspecto económico, el factor más importante es "tener un medio de subsistencia". Considerando que "ser emprendedor" es la carrera elegida, es apenas lógico que esperen que su empresa les aporte el sustento; sin embargo, esto no significa que sea un emprendimiento por necesidad en el sentido tradicional. Estos resultados coinciden con el hallazgo de Barba-Sánchez y Atienza-Sahuquillo (2012) quienes, producto de una revisión de literatura, encontraron que factores motivacionales del emprendedor como la necesidad de logro, la independencia, la afiliación, la competencia y el poder tienen mayor influencia en la decisión de iniciar un negocio que ganar dinero.

Podría decirse, entonces, que en la decisión de convertirse en emprendedores, predomina la motivación intrínseca, en el sentido de que esta se origina dentro del sujeto, a través de la auto-percepción como persona competente, eficaz y con determinación para actuar, o sea, es el individuo el agente causal de su propia actividad comportamental, tal como lo expuso Barberá-Heredia (2002). De acuerdo con Deci y Ryan (1985) esta motivación está muy relacionada con las necesidades. 
Por su parte los factores externos, más específicamente los que tienen que ver con las reglas de juego institucionales (North, 2006), como las políticas públicas, las campañas y organizaciones de fomento, y las fuentes de financiación, tienen poca influencia en la decisión de crear empresa para los emprendedores. Esto coincide con la afirmación de Shapero en cuanto a que el efecto principal del entorno político-legislativo-administrativo es negativo (Shapero, 1985); muestra de ello es la falta de acceso al mercado financiero en condiciones competitivas, que ha sido una problemática de larga data sin que se logre una solución de fondo.

Kantis et al. (2004) encontraron en su estudio que la mayoría de los emprendedores financiaron la creación de su empresa con ahorros personales y de la familia. Al respecto afirma que, no obstante las evidencias acerca de la importancia de la creación de empresas para el desarrollo económico, las nuevas empresas presentan dificultades para su consolidación y desarrollo, debido a dificultades en el acceso a financiamiento, tanto por el racionamiento de crédito y capital como por unas condiciones financieras muy desfavorables. Este es el caso para los emprendedores MIPES, quienes en su mayoría tuvieron que financiar la puesta en marcha de sus empresas con recursos propios o de sus familias.

En general, el aspecto económico es el que más golpea a los microempresarios, especialmente aquellos con empresas manufactureras, ya que requieren mayor capital de trabajo y el ciclo de recuperación es, por lo general, más largo que en las empresas de servicios. En este sentido, Sastre (2013) encontró que el emprendedor, a medida que se consolida, percibe que la falta de recursos financieros se convierte en el mayor obstáculo para sostener el emprendimiento. Esta es una situación común tanto en los países en vías de desarrollo como en los desarrollados (Moriano, Sánchez, \& Palací, 2004; Red española de equipos regionales, 2014). Es justamente esta realidad, la que hace que los factores personales, especialmente los más relacionados con motivación de logro sean los más importantes, pues como lo plantean Stewart y Roth (2007), son los individuos más decididos y persistentes los que logran crear, consolidar y mantener sus empresas.

El acompañamiento externo tampoco es en un gran motivador. Este hallazgo coincide con el de Barba-Sánchez et al. (2007), en cuanto a que las altas tasas de fracaso empresarial, especialmente cuando se trata de pymes en sus primeros años de vida, ponen en duda la validez de los programas de fomento de la creación de empresas. Sobre este aspecto, 
Leiva (2008) propone que estos programas tienden a considerar al emprendedor como un tomador de decisiones dentro de un ambiente económico, sin tener en cuenta que los atributos personales del individuo pueden ser un factor explicativo del proceso. En forma similar, Orrego (2008) explica que las universidades y algunas instituciones de carácter público y privado que ofrecen enseñanza en creación de empresas, basan su formación exclusivamente en esquemas funcionalistas y operativos, y propone una ruptura con la perspectiva economicista que permita reconocer al ser humano como portador de la voluntad de emprender y responsable de sus actos, con competencias para innovar e inmerso en la cultura. Otros autores recomiendan que estos programas se enfoquen primero en aumentar la autoeficacia empresarial (Segal, Borgia \& Schoenfeld, 2005).

En otros casos, las instituciones de fomento y sus programas sí logran que se creen empresas, pero tal vez porque no tienen en cuenta los aspectos motivacionales y personales, muchas de estas desaparecen rápidamente. De acuerdo con el informe GEM, la tasa de creación en Colombia y particularmente en Antioquia es de las más grandes a nivel mundial, pero así también la de cierre (GEM, 2014). De otro lado, aunque estos programas e instituciones no son tan eficaces promoviendo la creación de empresas, si lo son en etapas posteriores, como soporte para fortalecer las empresas. En este mismo sentido, Marulanda y Morales (2016) encontraron que para los emprendedores son de gran ayuda los programas como mentorías y ruedas de negocios, diseñados por entidades de fomento para el fortalecimiento de empresas en funcionamiento.

Si se considera que estas instituciones invierten una gran cantidad de recursos y esfuerzos en sus programas de acompañamiento, los incentivos deben ser encauzados hacia aquellos individuos que tengan un mayor potencial de desarrollar con éxito el proceso de creación y gestión de la empresa, por tanto es necesario crear mecanismos que permitan identificar las verdaderas motivaciones que mueven a las personas que solicitan el apoyo de dichas instituciones (Marulanda-Valencia, Montoya, \& Vélez, 2014b).

De igual forma la oportunidad, concebida como algo que se encuentra en el entorno y es percibido por algunos individuos que deciden explotarla, tampoco es un condicionante de la actividad emprendedora, mientras que en otras investigaciones como la de Kirkwood (2004) fue uno de los mayores motivadores para crear empresa. 
En cuanto a los factores que componen la dimensión "experiencia laboral", estos no tienen una importancia relativa tan alta; sin embargo, las situaciones categorizadas como: "inconformidad con la situación laboral cuando era empleado" (55 \%) y "terminación de vinculación laboral y oportunidad de continuar en empresa propia” (46\%) pueden convertirse en los factores de empuje (Shariff \& Saud, 2009; Rauch \& Frese, 2000; Carsrud \& Brännback, 2011), p como lo expone Sapero: "no hay nada como una dislocación personal para impulsar la acción”(Shaperto, 1985, p. 24).

A manera de síntesis, según el análisis de los resultados obtenidos en esta investigación a la luz de las teorías de Ajzen (1991) y Shapero (1985), el proceso de emprendimiento desde el individuo puede representarse con la figura 2. Según Shapero (1985), el emprendimiento se da cuando hay una percepción favorable de la conveniencia o deseabilidad, y de la viabilidad o factibilidad. Para el caso estudiado, el primer paso es la percepción de conveniencia y está dado por la valoración positiva de "ser emprendedor", que en Ajzen (1991) equivale a la actitud hacia el comportamiento. Los emprendedores han decidido que este es su camino de vida, su carrera, que les proporcionará una serie de satisfacciones de tipo personal, como el cumplimiento de un sueño, superar retos, alcanzar metas y, también, seguridad económica, lo cual se traduce en una valoración positiva del resultado esperado. Todo esto sumado al autoconcepto favorable sobre sus capacidades y habilidades emprendedoras, es un importante componente de la percepción de viabilidad o factibilidad, que además posibilita lo que es para Ajzen el control percibido del comportamiento, el cual permite la consolidación de la intención de crear empresa y en la la percepción de factibilidad externa no es determinante. Finalmente, cuando se presenta una circunstancia que Shapero considera "desestabilizante" y que puede ser positiva o negativa, ésta se convierte en la situación de empuje, que lleva a la realización del comportamiento, esto es, la creación y administración de la empresa. 


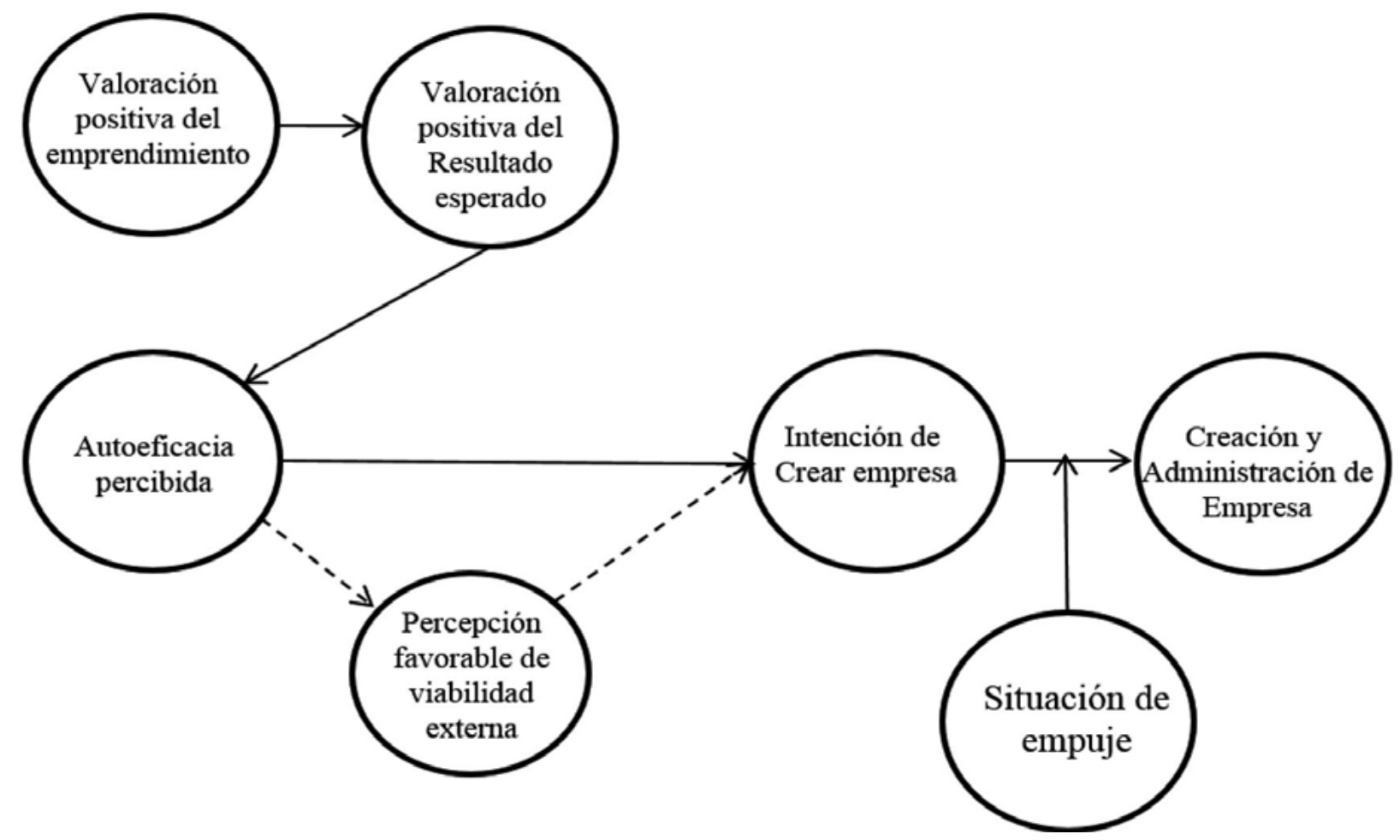

Figura 2. El individuo y su proceso de emprendimiento Fuente: Marulanda (2015).

\section{Conclusiones}

Considerando que de las seis dimensiones evaluadas, la relacionada con los aspectos personales es la más influyente y dentro de ésta, cumplir un sueño, alcanzar retos personales, el crecimiento personal, la autonomía e independencia y, en el aspecto económico, tener un medio de subsistencia, al integrar este resultado con el perfil descrito, especialmente en lo relacionado con la formación (60 \% profesionales, 37 \% tecnólogos y técnicos), se evidencia que no se trata de un emprendimiento por necesidad, sino que es la actividad que ellos escogieron como carrera y es apenas natural que esperen vivir de ella. Así, de de esta investigación se desprende que otro paradigma a superar es la clasificación tajante entre emprendimiento por necesidad y emprendimiento de oportunidad, la cual está muy arraigada en la literatura sobre el emprendimiento y que soporta muchos de los estudios empíricos. 
En relación con la disponibilidad de recursos, es muy influyente el factor "poner en práctica las habilidades emprendedoras que posee”. Este resultado, además de que corrobora la importancia de la autoeficacia percibida como motivación para la acción, permite entender que su preponderancia radica en que estas habilidades son las que garantizan el control sobre la actividad que van a desarrollar.

Los aspectos externos, compuestos por los programas de fomento y acompañamiento al emprendimiento, la financiación, la imagen y la influencia de amigos y familiares no son tan importantes. El factor que tiene una percepción más negativa es el de la financiación, debido a las limitaciones para acceder al mercado financiero, pues las pocas posibilidades de crédito se ofrecen en condiciones muy poco competitivas (tasas cercanas al $40 \%$ ), y es precisamente la falta de capital el principal problema que aqueja a los emprendedores.

La imagen ante la sociedad, así como la influencia de amigos y familiares, no son tan relevantes para los emprendedores. Antioquia ha sido una región reconocida nacional e internacionalmente como emprendedora, así que es apenas lógico que las iniciativas emprendedoras tengan buena aceptación social, y por tanto no es un asunto que preocupe a quienes desean dedicarse a esta actividad, pues para ellos es, además, una forma de contribuir con la sociedad generando empleo y suministrando sus productos y servicios. Respecto a la poca influencia de amigos y familiares, es una muestra más del deseo de independencia como motivación y como característica de los emprendedores.

Se cumple entonces el propósito general de esta investigación, en cuanto a destacar la importancia del individuo dentro del proceso emprendedor, con unas motivaciones más de carácter personal que económicas y de status. Los planteamientos teóricos desde las ciencias sociales permiten vislumbrar la complejidad del ser humano en sí y por tanto de los procesos en los que se encuentra inmerso, más cuando se trata de algo tan trascendente en su vida como la elección de carrera a la cual le dedica un gran porcentaje de su energía y sus recursos en lo que es para ellos el cumplimiento de un sueño.

Este trabajo también puede servir como base para diseñar un instrumento que permita identificar a aquellas personas que tienen una verdadera motivación para emprender, y por tanto el potencial para desarrollar en forma exitosa su negocio, y de esta manera, encauzar los recursos y esfuerzos al acompañamiento de éstas y sus proyectos. 


\section{Referencias}

Ajzen, I. (1991). The Theory of Planned Behavior. Organizational Behavior And Human Decision Processes 50, 179-211.

Autio, E., \& Kauranen, I. (1994). Technologist-Entrepreneurs Versus Non-Entrepreneurial Technologists: Analysis of Motivational Triggering Factors. Entrepreneurship \& Regional Development, 6(4), 315-328.

Bandura, A. (1977). Self-efficacy: Toward a Unifying Theory of Behavioral Change. Psychological Review, 84(2), 191-215.

BarbaSanchez, V., \& AtienzaSahuquillo, C. (2012). Entrepreneurial Behavior: Impact of Motivation Factors on Decision to Create a New Venture. Investigaciones Europeas de Dirección y Economía de la Empresa, (18), 132-138.

Barba-Sánchez, V., Atienza-Sahuquillo, C., Jiménez-Zarco, A. I., \& Martínez-Ruiz, M. P. (2007). Efectos de la motivacion y de la experiencia del empresario emprendedor en el crecimiento de la empresa de reciente creación. Recuperado de http://www. escp-eap. net/conferences/marketing/2007_cp/Materiali/Paper/Fr/BarbaSanchez_JimenezZarco_MartinezRuiz. pdf. España

Barberá-Heredia, E. (2002). Modelos explicativos en Psicología de la Motivación. Revista Electrónica de Motivación y Emoción 5(10), 6.

Carsrud, A., \& Brännback, M. (2011). Entrepreneurial Motivations: What Do We Still Need to Know? Journal of Small Business Management 49(1), 9-26.

Carter, N. M., Gartner, W. B., Shaver, K. G., \& Gatewood, E. J. (2003). The Career Reasons of Nascent Entrepreneurs. Journal of Business Venturing 18, 13-39.

Deci, E. L., \& Ryan, R. M. (1985). Intrinsic Motivation and Self-determination. New York: Plenum.

Edelman, L., Brush, C., Manolova, T., \& Greene, P. (2010). Start-up Motivations and Growth Intentions of Minority Nascent Entrepreneurs. Journal of Small Business Management 48(2), 174-196.

Farmer, S. M., Yao, X., \& Kung-Mcintyre, K. (2011). The Behavioral Impact of Entrepreneur Identity Aspiration and Prior Entrepreneurial Experience. Entrepreneurship Theory And Practice, 35(2), 245-273.

García, E. E. (1992). Incidencia de las motivaciones personales en el proceso de creación de la nueva PYME industrial. Revista Europea de Dirección y Economía de la Empresa, 1(1), 67-78.

GEM Colombia. (2014). Dinámica empresarial colombiana. Atelier 33. 
Hofstede, G. (1983). The Cultural Relativity of Organizational Practices and Theories. Journal of International Business Studies, 14(2), 75-89.

Kantis, H., Angelelli, P., \& Moori, V. (2004). El Desarrollo Emprendedor. América Latina y la experiencia Internacional. Washington: Banco Interamericano de Desarrollo Fundes Internacional.

Kirkwood, J. (2004). One Size Doesn't Fit All: Gender Differences in Motivations for Becoming an Entrepreneur (tesis doctoral), Universidad de Otago, Dunedin, Nueva Zelanda.

Leiva, J. C. (2008). Nacen empresas de las actividades de fomento al espíritu emprendedor? TEC Empresarial, 2(1), 16-27.

Manolova, T., Brush, C., \& Edelman, L. (2008). What Do Women Entrepreneurs Want? Strategic Change, 17(3-4), 69-82.

Mariano, J.A., Trejo, E., \& Palací, F. (2001). Perfil psicosocial del emprendedor. Revista de Psicología Social, 16(2), 229-242.

Marulanda, F. Á. (2015). Una contribución a la comprensión de las motivaciones del emprendedor y su conexión con el emprendimiento MIPES de Valle de Aburrá. Medellín: Universidad Nacional de Colombia.

Marulanda, F. Á, \& Morales, S. T. (2016). Entorno y motivaciones para emprender. Revista EAN, (81), 12-28.

Marulanda, F. Á., Montoya, I. A., \& Vélez, J. M. (2014 B). Teorías motivacionales en el estudio del emprendimiento. Pensamiento E Gestión, (36), 206-238.

Maslow, A. (1991). Motivación y Personalidad. Madrid: Ediciones Díaz de Santos.

McClelland, D. (1961). The Achieving Society. New York: A Free Press Paperback.

Morales-Gualdrón, S., Gutiérrez-Gracia, A., \& Roig-Dobón, S. (2009). The Entrepreneurial Motivation in Academia: A Multidimentional Construct. International Entrepreneurship and Management Journal, 5(3), 301-317.

Moriano, J. A., Sánchez, M. L., \& Palací, F. J. (2004). Un estudio descriptivo sobre los emprendedores en España, la República Checa y Bulgaria. En Congreso de Valencia, Valencia, España.

North, D. C. (2006). Instituciones, Cambio Institucional y Desempeño Económico. México: Fondo de Cultura Económica.

Orrego, C. (2008). La dimensión humana del emprendimiento. Revista Ciencias Estratégicas, 16(20), 225-235.

Paturel, R. (1997). Pratique du management straégique. Grenoble: Presses Universitaires de Grenoble. 
Quevedo, L. M., Izar, J. M., \& Romo, L. (2010). Factores endógenos y exógenos de mujeres y hombres emprendedores de España, Estados Unidos y México. Investigación y Ciencia, 18(46), 57-63.

Rauch, A., \& Frese, M. (2000). Psychological Approaches to Entrepreneurial Success. A General Model and an Overview of Findings. En C. Cooper, \& I. Robertson, International Review of Industrial and Organizational Psychology (pp. 101-142). Chichester: Wiley.

Red española de equipos regionales. (16 de 6 de 2014). Global Entrepreneurship Monitor. Recuperado de http//www.cise.es/wp-content/uploads/INFORME-GEM-ESPAÑA-20131.pdf

Rodríguez, A. (2009). Nuevas perspectivas para entender el emprendimiento empresarial. Pensamiento y gestión, (26), 94-119.

Sastre, R. (2013). La motivación emprendedora y los factores que contribuyen con el éxito del emprendimiento. Ciencias Administrativas, 1, 1-10.

Segal, G., Borgia, D., \& Schoenfeld, J. (2005). The Motivation to Become an Entrepreneur. International journal of Entrepreneurial Behavior \& research, 11(1), 42-57.

SENA. (2012). Lineamientos generales para el grupo de Emprendimiento, Empresarismo y Fondo Emprender. Recuperado de http//www.http://compromiso.sena.edu.co/documentos/docs_pdf/1391445796_GEE-P-003_Procedimiento_Gestion_para_el_Fortalecimiento_ Empresarial.xlsx.pdf

Shane, S., \& Venkataraman, S. (2000). The Promise of Entrepreneurship as a Field of Research. Academy of Management Review, 25(1), 217-226.

Shane, S., Locke, E. A., \& Collins, C. J. (2003). Entrepreneurial Motivation. Human Resource Management Review, 13, 257-279.

Shapero, A. T. (1985). The Entrepreneurial Event. Ohio: College of Administrative Science, Ohio State University.

Shariff, M. N., \& Saud, S. M. (2009). An Attitude Approach to the Prediction of Entrepreneurship on Students at Institution of Higher Learning in Malaysia. International Journal of Business and Management, 4(4), 129-135.

Shepherd, D. (2005). Prior Knowledge, Potential Financial Reward, and Opportunity Identification. Entrepreneurship Theory and Practice, 29(1), 91-112.

Stewart, W., \& Roth, P. (2007). A Meta-Analysis of Achievement Motivation Differences between Entrepreneurs and Managers. Journal of Small Business Management 2007 45(4), 401-421.

Varela, R., \& Bedoya, O. (2006). Modelo conceptual de desarrollo empresarial basado en competencias. Estudios Gerenciales, 22(100), 21-47. 